\title{
Mozart Effect in Musical Fit? A Commentary on Yeoh \& North
}

\author{
EMERY SCHUBERT \\ Empirical Musicology Group, University of New South Wales
}

\begin{abstract}
Musical fit, the congruence between music and product leading to improved response, is presented as an explanation of differences in recall of food items from two cultures. Musical fit predicts that, in this case, more Indian food items would be recalled when Indian music was playing, and more Malay food items would be recalled when Malay music was played. The underlining premise of this prediction is priming (the music primes memories of associated food items, facilitating recall). The testing was performed using three ethnic groups of participants, Indian, Malay, and Chinese. The priming effects were only identified among the Chinese group. A reanalysis of the data presented here indicates that the overall recall of food items is best facilitated by Indian music, regardless of whether the food item is Indian or Malay, and further, each ethnicity reported more items in the Indian music condition. The Mozart effect was also based on the premise of priming activation and was later debunked in favor of an arousal-mood hypothesis. This commentary discusses whether arousal-mood, a more generalized activation leading to improved performance than priming, might better explain the results. While priming and congruence more plausibly explains musical fit than the Mozart effect, the possibility of testing other hypotheses are considered worthwhile.
\end{abstract}

Submitted 2010 March 8; accepted 2010 March 12.

KEYWORDS: musical fit, priming, arousal-mood, Mozart effect

IN consumer research 'musical fit' is an expression that has come to mean the congruence between a service/product being promoted and the music played during the promotion. In other words, the music 'complements' (MacInnis \& Park, 1991) the product. A high fit refers to the (probably) culturally determined match between product and music. This has been attributed to increasing consumer-like behaviors, such as longer shopping time, purchasing more items/services, and the individuals having positive evaluations about matters concerned with the consumer experience. Zander (2006) provides an example of the, at times, speculative nature of the fit:

"either a rock song or a classical work could be used in a commercial for a car, which is considered as a typical high involvement product ... . The rock song would probably underline consumers' beliefs about the power, speed and competitiveness of the car, whereas the classical piece might emphasize beliefs about its interior trim, luxury and elegance. According to the idea of musical fit, both versions would make sense in their aim to transport relevant information about the car because both are congruent." (p. 467468).

A simpler example is Jacob's (2006) finding that drinking songs played in a bar appeared to increase the amount of time and amount of money spent in that bar compared to when Top 40 or cartoon music was being played.

In cognitive psychology literature, musical fit also falls under the banner of auditory priming (as pointed out by Yeoh \& North, (2009)), where music is used to help encode and later facilitate recall of a message, or - in the absence of any explicit earlier pairing - that a happy coincidence of congruent matching is discovered (as might be the case with the classical music and the luxury of the car interior). More interestingly, some literature views music as having potential for affective priming. For example, Sollberge, Rebe, and Eckstein, (2003) found that consonant chords led to faster evaluation of positive valenced words 
such as 'miracle' than when a dissonant chord was presented. The effect was lost when the affective priming was mismatched with the word, such as a dissonant chord with 'holiday', or consonant chord with 'war'. The principle of congruence is therefore, obviously, at play here, too. Affective priming suggests that there is more to musical fit than simple association.

Yeoh and North (2010) take the idea of musical fit one step further, by testing whether people of different cultures experience enhanced recall when music is congruent with the items to be recalled. Given that congruence may well be an important factor in the musical fit effect, and that this congruence is probably learnt through enculturation, the study is a particularly interesting one. It examines whether culturally specific congruence can also influence people from different cultures. To this end, Yeoh and North assembled three groups of participants of separate and distinct ethnicities: Malay, Indian, and Chinese. Individuals listened to either Malay or Indian music (which I will refer to as an intervention), and were asked to report as many Malay or Indian food items as they could. A musical fit hypothesis that makes no cross-cultural claims would predict that more Indian food items will be reported by the Indian ethnic group when the musical intervention is Indian than when the intervention is Malay (because, presumably, people from an Indian culture are likely to have had associations made between Indian products such as food and music). Similarly, more Malay food items will be recalled by the Malay ethnic group when Malay music is played. The results reported by Yeoh and North confirm this hypothesis for the Indian ethnic group. When they listened to Indian music they recalled 12.38 (SD = 6.18) Indian food items, compared to 7.96 (2.91) Indian food items when Malay music was played (Cohen's d $=0.915) .{ }^{[1]}$

However, the results for the Malay group was in opposition to this musical fit hypothesis, with more Malay items recalled (on average) when Indian music was played $(9.50$ items, SD $=4.17$ ) than when Malay music was played $(7.87$ items, $S D=2.95)$. The effect size in the 'wrong' direction, as measured by Cohen's d (0.451), was reasonably strong. Despite this negative result, Yeoh and North did report an interesting cross-cultural effect, with the ethnic Chinese group appearing to be affected by the congruence of the music and recall: on average, more Malay food items were recalled with Malay music than Indian music, and more Indian food items were recalled for the Indian music than Malay music.

In the context of musical fit and its associated congruence hypotheses, the results are mixed. Musical fit provides one explanation of the Indian ethnic group responses, and it suggests that crosscultural absorption has taken place such that other cultures (in this case, by those of Chinese ethnicity) can be sensitive to the fit effect. Thus, we could conclude that individuals do not need to have a strong personal connection or identify with the food items and music to be able to process them congruently, or for one to prime the other. This is one explanation of the results.

The study is indeed an interesting one in terms of its design and results. However, in this commentary I raise the possibility that a better explanation of the results could be provided than the priming effect posited or implied by the musical fit hypothesis. I will argue that an alternative may explain the two positive musical fit hypothesis results (for the Indian and Chinese ethnicity groups), but also the negative result of the Malay ethnicity group. I do this by calling forth, and drawing some parallels with, the historical stages of the debate evoked by the 'Mozart effect'.

\section{MOZART EFFECT EXPLANATIONS APPLIED TO MUSICAL FIT}

The Mozart effect refers to an increased score in spatial-temporal reasoning tasks when listening to certain kinds of stimuli (such as music by Mozart). The explanations of this effect that dominated the literature throughout the early 1990s was a priming based explanation and was presented by the pioneer of the effect, Frances Rauscher and her colleagues (Rauscher, Shaw, \& Ky, 1995). The argument posited that neural circuits activated when listening to Mozart were in the vicinity of those circuits used for spatial-temporal and other processing, and therefore provided priming activation for these other functions: "Music acts as an 'exercise' for exciting and priming the common and sequential flow of the cortical firing patterns responsible for higher brain functions." (p. 47).

This mechanism also provides a neural explanation of the priming-based musical fit hypothesis: The Indian Music stimulates and excites semantically associated networks, and so connections more closely associated with Indian music_-such as Indian food-would be primed, hence facilitating better recall of the Indian food items. This explanation is founded in the classic semantic-network theory of Collins and Loftus (1975) and ties in with Yeoh and North's 'ceiling effect' explanation of some of their 'weaker' results: the participants of Malay ethnicity recalled four to five Indian food items regardless of the 
music intervention, suggesting that they were not aware of many Indian food items to begin with; there are no more Indian food items to be activated. In other words, a ceiling level had been reached. The priming musical fit explanation can, therefore, explain this result, albeit in a weak way.

But the priming-based musical fit hypothesis does not explain why recall increased for Indian music for non-Indian food items. Most peculiar in this respect is the increase in mean number of recalled Malay food items by the Malay group when Indian music was played $(9.50$ items, SD $=4.17$ compared with 7.87 items, SD = 2.95 for Malay music intervention by the Malay ethnicity group) because, presumably, the Malay items would not have been primed by the Indian music. Despite being in opposition to the priming hypothesis, the effect size according to a simple Cohen's d calculation was reasonably strong (0.451).

\section{AROUSAL-MOOD HYPOTHESIS}

An alternative hypothesis that I will consider draws on the next stage of the history of the Mozart effect literature, which was ignited by the failure of the effect to be replicated under certain conditions, the failure of the priming explanation. In short, several reports were emerging that improved spatial-temporal reasoning scores could be achieved by means other than playing Mozart. Of the numerous variables and stimuli investigated (for a review, see Husain, Thompson, \& Schellenberg, 2002), better predictors of the improved spatial reasoning task scores emerged that were concerned with preference, arousal and mood, rather than priming (Nantais \& Schellenberg, 1999; Thompson, Schellenberg, \& Husain, 2001). The new, dominating hypothesis that emerged to explain the Mozart effect from the late 1990s was coined the 'arousal-mood' hypothesis.

Specifically, the arousal-mood hypothesis suggests that when the music makes individuals feel happy and aroused (active) they will be more engaged with the task. The hypothesis has its roots in optimal arousal theory (Bower, 1992; Schreter, 1990). From an evolutionary perspective, it makes sense to focus on information in the environment with a greater level of engagement when level of arousal (for example, as a result of threat or opportunity) is at a high level, but not so high that the organism is debilitated. In music cognition research, the argument has emerged that music which is both enjoyed (improves mood) and increases arousal (for example, a piece that you like, that has a fast tempo), will therefore facilitate better cognitive functioning, including performance in spatial-temporal reasoning and recall tasks. Husain et al. (2002, p. 154) surmise the contrast between arousal-mood and priming explanations: "listening to music may indeed be associated with subsequent cognitive abilities, but the route is probably mediated by arousal and mood rather than one of direct influence". Additionally, the arousal-mood hypothesis suggests that the intrinsic properties of the music (whether Mozart, Indian classical, or Malay instrumental) are not crucial factors, but the experiences that the individual has with the music (such as familiarity, arousal and preference) are crucial.

This may seem contradictory. Surely the relationship a person has with a piece of music bears at least some similarities with the intrinsic properties of the music: Fast music can be classified as fast more or less objectively, for example. But it is also the case that arousal-mood responses will be a function of properties that are subjective, such as familiarity and preference (North \& Hargreaves, 1997; Rickard, 2004). Indeed, there may be an interaction. Preferred, fast tempo music is, all things being equal, going to produce a higher happy-aroused rating than unfamiliar, fast music, but also, by the same token, familiar slow music. Again, this is regardless of whether the music is Mozart, Indian, Malay or the Beatles.

The arousal-mood hypothesis, then, predicts that for the Yeoh and North data, music that is familiar/preferred and more arousing will lead to more items being recalled regardless of the recall task (Malay or Indian food items). Although detailed musical characteristics of the pieces were not reported, Yeoh and North indicated that one piece had a 'moderate tempo' and the other a 'somewhat moderate speed'. For now let us assume that the tempo was the same. If the arousal-mood hypothesis were supported it would mean that overall preference and familiarity (Schubert, in press) with Indian music (whether the style or the piece) were higher than the Malay instrumental pieces. Recall for all food items increased when Indian music was played for both Malay and Indian ethnic groups. Since data were not present for these kinds of variables I can only speculate, based on the arousal-mood hypothesis, that both Indian and Malay participants had more positive and/or arousing responses to the Indian classical music piece that the Malay music. It may also be worth doing a closer analysis of sources of differentiation in arousal-mood between the two sets of interventions that go beyond a general tempo description. 
Even so, the lynchpin that makes the arousal-mood hypothesis a more persuasive account of the data than priming is the result of the Malay ethnic group, mentioned earlier. But does arousal-mood explain the responses of the participants of Chinese ethnicity, which the priming account does explain? The clearest difference across conditions for the Chinese group was the Indian food item count in the Indian music intervention, which increased from a mean of 3.96 items $(\mathrm{SD}=2.03)$ to 5.54 items $(\mathrm{SD}=2.43)$ with a Cohen's $d$ of 0.706 . The number of Malay food items recalled when Malay music was played had a mean of $5.12(\mathrm{SD}=2.49)$, which turns out to be a relatively small increase from 4.67 items $(\mathrm{SD}=2.08)$ when Indian music was played (Cohen's $d=0.196$, a small effect size). Hence, after analyzing various pairs of cells from Table 1 of Yeoh and North, it becomes evident that Indian music is the dominant factor related to general recall, rather than a music congruent response. If the arousal-mood hypothesis could be tested, it might prove to be better explanation of the effects. In the absence of data that allows direct testing of the arousal-mood hypothesis, the prediction that this alternative hypothesis would make of the data set is that the Indian classical music used in the study was, in some combination, more enjoyed and more arousing than the Malay music examples used.

\section{CONCLUSIONS}

In this commentary, I have provided a possible alternative, and perhaps superior explanation of the data than that offered by musical fit and its assumption of operating through priming. I do not, however, view one hypothesis as replacing another. It may be that the mechanisms that explain both hypotheses are in operation. The interest, then, is to see if one hypothesis provides better explanations of data than others under various conditions. In the current example, musical fit may not provide the best explanation of the data. Further research is required to determine if arousal-mood or some other hypothesis provides a better explanation of the effects found in the paper under scrutiny, or of a similar set of experimental circumstances. It is also worth noting that cognitive or affective priming provides a legitimate potential explanation for increased recall, compared to the same framework applied to the Mozart effect because we are not, here, dealing with priming in (probably) unrelated domains (music to spatial-temporal reasoning), but congruent cross-domain links (such as Malay music to Malay food items). It may therefore be more difficult to dislodge musical fit literature from its priming assumptions, and rightly so. But the possibilities of several mechanisms operating in tandem or multitude underlie a more complex, yet complete understanding of the operation of human mind and memory. The Yeoh and North article is to be applauded for its neatness in design, but also for provoking further thought on possible explanations of musical fit as a result of the innovation of exploring cross-cultural behaviors.

\section{NOTES}

[1] I report Cohen's d without adjustment even though I apply them to multiple tests, which makes them, in a strict sense, not the best statistic to use. However, what will become important here is the ranking of these effect size estimates.

\section{REFERENCES}

Bower, G.H. (1992). How might emotions affect learning? In S.-Å. Christianson (Ed.), The handbook of emotion and memory: Research and theory (pp. 3-32). New Jersey: Lawrence Erlbaum Associates.

Collins, A.M., \& Loftus, E.F. (1975). Spreading activation theory of semantic processing. Psychological Review, 82(6), 407-428.

Husain, G., Thompson, W.F., \& Schellenberg, E. (2002). Effects of musical tempo and mode on arousal, mood, and spatial abilities. Music Perception, 20(2), 151-171. 
Jacob, C. (2006). Styles of background music and consumption in a bar: An empirical evaluation. International Journal of Hospitality Management, 25(4), 716-720.

MacInnis, D.J., \& Park, C.W. (1991). The differential role of characteristics of music on high-and lowinvolvement consumers' processing of ads. Journal of Consumer Research, 18, 161-173.

Nantais, K.M., \& Schellenberg, E.G. (1999). The Mozart effect: An artifact of preference. Psychological Science, 10(4), 370-373.

North, A.C., \& Hargreaves, D.J. (1997). Experimental aesthetics and everyday music listening. In D.J. Hargreaves \& A.C. North (Eds.), The social psychology of music (pp. 84-103). Oxford: Oxford University Press.

Rauscher, F.H., Shaw, G.L., \& Ky, K.N. (1995). Listening to Mozart enhances spatial-temporal reasoning: towards a neurophysiological basis. Neuroscience letters, 185(1), 44-47.

Rickard, N.S. (2004). Intense emotional responses to music: A test of the physiological arousal hypothesis. Psychology of Music, 32(4), 371-388.

Schreter, Z. (1990). Connectionism: A link between psychology and neuroscience? In J. Stender \& T. Addis (Eds.), Symbols versus neurons? (pp. 152-203). Netherlands: IOS.

Schubert, E. (in press). Affective, evaluative, and collative responses to hated and loved music. Psychology of Aesthetics, Creativity, and the Arts.

Sollberge, B., Rebe, R., \& Eckstein, D. (2003). Musical chords as affective priming context in a wordevaluation task. Music Perception, 20(3), 263-282.

Thompson, W.F., Schellenberg, E.G., \& Husain, G. (2001). Arousal, mood, and the Mozart effect. Psychological Science, 12(3), 248-251.

Yeoh, J.P.S., \& North, A.C. (2009). The effects of musical fit on choice between competing pairs of cultural products. Empirical Musicology Review, 4(4), 130-133.

Yeoh, J.P.S., \& North, A.C. (2010). The effects of musical fit on consumers' ability to freely recall related products. Empirical Musicology Review, 5(1), 2-8.

Zander, M.F. (2006). Musical influences in advertising: How music modifies first impressions of product endorsers and brands. Psychology of Music, 34(4), 465-480. 